\title{
Noniterative Data-driven Controller Tuning Using the Correlation Approach
}

\author{
Alireza Karimi, Klaske van Heusden and Dominique Bonvin
}

\begin{abstract}
Data-driven controller tuning for model reference control problem is investigated. A new controller tuning scheme for linear time-invariant single-input single-output systems is proposed. The method, which is based on the correlation approach, uses a single set of input/output data from openloop or closed-loop operation. A specific choice of instrumental variables makes the correlation criterion an approximation of the model reference control criterion. The controller parameters and the correlation criterion are asymptotically not affected by noise. In addition, based on the small gain theorem, a sufficient condition for the stability of the closed-loop system is given in terms of the infinity norm of a transfer function. An unbiased estimate of this infinity norm can be obtained as the solution to a convex optimization problem using an infinite number of noise-free data. It is also shown that, for noisy data, the use of the correlation approach can improve significantly the estimate. The effectiveness of the proposed method is illustrated via a simulation example.
\end{abstract}

\section{INTRODUCTION}

Suppose that a set of input/output data from open-loop or closed-loop process operation is available and the objective is to design a low-order controller that minimizes the twonorm of the difference between a given reference model and the closed-loop system. The classical model-based approach suggests a three-step procedure : (1) identify a plant model and validate it using the data, (2) compute a high-oder controller that minimizes the criterion and stabilizes the plant model, (3) reduce the controller order by standard controller order reduction techniques. These three steps include essentially some optimization algorithms and iterative methods for model identification and validation procedures. Data-driven controller tuning approaches try to lump these three steps and present a direct "data-to-control" algorithm. They typically use parameter estimation algorithms to estimate the controller parameters by minimizing a control criterion. These approaches are particularly interesting when a mathematical model of the plant is not available and/or the nonlinear behavior of the plant cannot be identified easily and considered for controller design.

Data-driven approaches for controller tuning have been an attracting research subject for a long time. The first publications appeared in the 60s in the context of dual control and adaptive control. A survey of these approaches can be found in [1]. In recent years, a revival of interest in this subject has been observed in the control community by the

The authors are with the Laboratoire d'Automatique of Ecole Polytechnique Fédérale de Lausanne (EPFL), 1015 Lausanne, Switzerland.

This research work is financially supported by the Swiss National Science Foundation under Grant No. 200020-105139.

Corresponding author: alireza.karimieepfl.ch introduction of Iterative Feedback Tuning (IFT) [2], [3]. IFT is a model-free approach that iteratively minimizes a quadratic cost using several closed-loop experiments. The main feature of this algorithm is that an unbiased estimate of the gradient of the cost function can be obtained from a specific closed-loop experiment in each iteration. A similar approach, Iterative Correlation-based Tuning (ICbT), minimizes the cross-correlation function between the closed-loop error and the reference signal [4]. This way, the controller parameters are asymptotically not affected by noise. Virtual Reference Feedback Tuning (VRFT) converts the model reference control problem to an identification problem [5]. It is shown that with a specific data filter an approximation of the model reference control criterion can be minimized [6]. Iterative Controller Unfalsification (ICU) tries to minimize the infinity norm of the model reference control criterion [7]. In each iteration, a controller is designed that cannot be falsified by the data from the same and previous iterations. The main problem is that the approach considers only normbounded disturbances and stochastic properties of the noise are not taken into account. This leads to underestimation of the control criterion and therefore to unfalsification of the controllers that do not meet the specifications.

In model-based approaches, the stochastic disturbances are treated in the model identification and validation step by some asymptotic analyses, while the problem of robust stability is considered in the second and third step. The main challenges in data-driven approaches consists of considering in one step the problem of robust stability and the effect of stochastic disturbances on the controller parameters. Moreover, investigation of controller robustness typically involves a nominal plant model and uncertainties that cannot be effectively considered in model-free algorithms.

In this paper, a new algorithm for controller tuning based on the correlation approach is proposed. A tuning scheme is introduced that convexifies the data-driven optimization related to the model reference control problem and uses a single set of data to evaluate the control criterion. A correlation approach based on the extended instrumental variable technique is used to estimate the controller parameters. Furthermore, a data-driven stability test is introduced that falsifies the destabilizing controllers before applying them to the real plant. This test takes advantage of the correlation technique and can also be used for other datadriven controller tuning methods with a model reference criterion.

The proposed approach has the same asymptotic properties as the VRFT technique for linear systems. However, there 


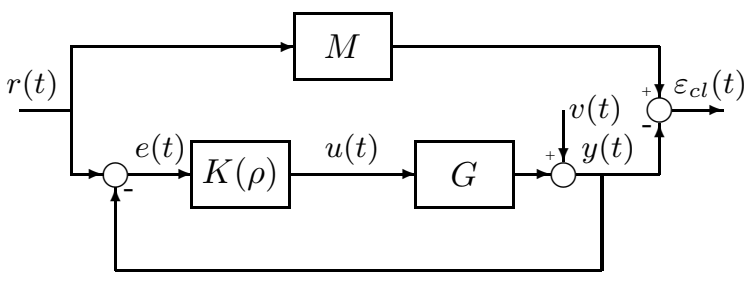

Fig. 1. Model reference control problem

is no need to introduce virtual signals nor to compute the inverse of the reference model. For noisy data, a single set of data is sufficient thanks to the correlation approach. Moreover, with a finite number of data, the presented approach is less sensitive to noise and leads to more robust controllers. It should be mentioned that the VRFT approach can be applied to nonlinear systems [8], which is not the case for the proposed approach because the controller and plant are swapped in the tuning scheme.

The paper is organized as follows. Section II introduces a new controller tuning scheme that can be used for open-loop shaping and for model reference control problems. The use of the correlation approach and the choice of instrumental variables are discussed in Section III. A controller (in)validation test based on a convex optimization algorithm is given in Section IV. Section V compares the proposed method with VRFT via a simulation example. It is also shown that destabilizing controllers can effectively be detected by the proposed approach. The paper ends with some concluding remarks.

\section{Controller Tuning Scheme}

Suppose that a set of input/output noisy data from openloop or closed-loop operation of a single-input single-output linear time-invariant discrete-time system is available. Let the output of the system be generated by the model :

$$
y(t)=G\left(q^{-1}\right) u(t)+v(t)
$$

where $y(t)$ is the plant output, $u(t)$ the plant input, $v(t)$ a zero-mean noise signal and $G\left(q^{-1}\right)$ the unknown plant model with $q^{-1}$ the backward shift operator. The objective is to compute the parameters $\rho$ of a linear discrete-time controller $K\left(\rho, q^{-1}\right)$ for $G\left(q^{-1}\right)$ such that the closed-loop output follows the output of a reference LTI model $M\left(q^{-1}\right)$ as well as possible. This can be achieved by minimizing the two-norm of the difference between the reference model and the achieved closed-loop system. Fig. 1 shows the block diagram of the model reference control problem. The control criterion is defined as :

$$
J_{m r}(\rho)=\left\|M-\frac{K(\rho) G}{1+K(\rho) G}\right\|_{2}^{2}
$$

Minimizing this criterion is a standard control problem when the plant model $G$ is known. In a data-driven approach, the two-norm of the closed-loop error $\varepsilon_{c l}(t)$ is minimized for a random white noise reference signal. Apart from the effect of noise and possible instability of the closed-loop system,

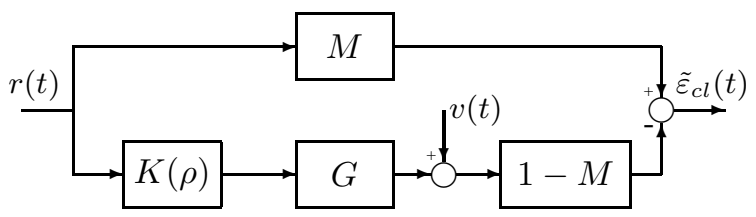

Fig. 2. Approximation of model reference control problem leading to a convex criterion

which are common problems in all data-driven approaches, the model reference control problem has two additional difficulties :

- The criterion (2) is not convex with respect to the controller parameters $\rho$. Therefore, only a local minimum of the criterion can be obtained.

- Each evaluation of the criterion requires an experiment on the real system, which leads to an iterative algorithm.

These problems are present in IFT [2] and ICbT [4] that are both iterative and guarantee only local convergence of the criterion. In order to fix the first problem, the difference between the desired loop transfer function and the achieved one can be minimized. Let $M\left(q^{-1}\right)$ be expressed as :

$$
M\left(q^{-1}\right)=\frac{K^{*}\left(q^{-1}\right) G\left(q^{-1}\right)}{1+K^{*}\left(q^{-1}\right) G\left(q^{-1}\right)}
$$

where $K^{*}\left(q^{-1}\right)$ is the ideal controller. The desired looptransfer function $K^{*}\left(q^{-1}\right) G\left(q^{-1}\right)$ becomes :

$$
K^{*}\left(q^{-1}\right) G\left(q^{-1}\right)=\frac{M\left(q^{-1}\right)}{1-M\left(q^{-1}\right)}
$$

which gives :

$$
\frac{1}{1+K^{*}\left(q^{-1}\right) G\left(q^{-1}\right)}=1-M\left(q^{-1}\right)
$$

The term $1 /(1+K(\rho) G)$ in (2) can be approximated by $1 /\left(1+K^{*} G\right)=1-M$, which allows defining a new criterion :

$$
J(\rho)=\|W[M-K(\rho) G(1-M)]\|_{2}^{2}
$$

where $W$ is an appropriate weighting filter which will be computed subsequently such that criterion $J(\rho)$ becomes a good approximation of the model reference criterion $J_{m r}(\rho)$. The criterion $J(\rho)$ is convex with respect to the controller parameters if $K(\rho)$ is parameterized linearly in $\rho$. Moreover, if $K^{*}$ is in the set of parameterized controllers, it will be the minimizer of $J(\rho)$. This criterion can be minimized by minimizing the two-norm of the noise-free part of $W\left(q^{-1}\right) \tilde{\varepsilon}_{c l}(\rho, t)$ where

$$
\tilde{\varepsilon}_{c l}(\rho, t)=[M-K(\rho) G(1-M)] r(t)-(1-M) v(t)
$$

with $r(t)$ being the reference signal (see Fig. 2). However, as mentioned before, several experiments on the real system are needed to evaluate this criterion. This difficulty can be eliminated by changing the place of the controller and putting it after the filter $1-M$ as shown in Fig. 3. The main advantage of this scheme is that an estimate of the control 


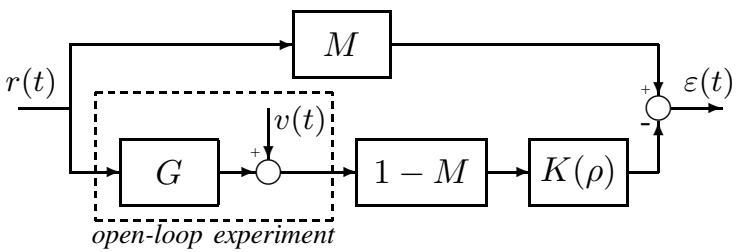

Fig. 3. Approximation of model reference control problem making use of a single open-loop experiment

criterion (6) can be minimized with a single experiment on the system. Suppose that the system $G$ is excited in open loop with $u(t)=r(t)$ and the noisy output $y(t)$ is measured. From this experiment, the error signal $\varepsilon(\rho, t)$ can be expressed as a function of the controller parameters $\rho$ :

$$
\begin{aligned}
& \varepsilon(\rho, t)=M r(t)-(1-M) K(\rho) y(t) \\
& =[M-G(1-M) K(\rho)] r(t)-(1-M) K(\rho) v(t)
\end{aligned}
$$

It is clear that, in absence of noise $(v(t) \equiv 0), \varepsilon(\rho, t)$ is equal to $\tilde{\varepsilon}_{c l}(\rho, t)$. In presence of noise, the correlation approach can be used to reduce the effect of noise on the criterion and the controller parameters.

\section{Controller Tuning Using the Correlation APPROACH}

It is clear from the criterion (6) that the ideal controller $K^{*}=K\left(\rho^{*}\right)$ leads to $M=K\left(\rho^{*}\right) G(1-M)$ and the error signal $\varepsilon\left(\rho^{*}, t\right)$ in $(8)$ becomes filtered noise :

$$
\varepsilon\left(\rho^{*}, t\right)=-(1-M) K\left(\rho^{*}\right) v(t) .
$$

Since $v(t)$ is not correlated with the reference signal $r(t)$, the error signal for the ideal controller will not be correlated with the reference signal either. Therefore, the main idea is to tune the controller parameters $\rho$ such that $\varepsilon(\rho, t)$ is uncorrelated with the reference signal.

\section{A. Correlation Approach}

To proceed, let the correlation function be defined as :

$$
f(\rho)=E\left\{\zeta_{w}(t) \varepsilon(\rho, t)\right\}
$$

where $E\{\cdot\}$ is the mathematical expectation and $\zeta_{w}(t)$ a vector of instrumental variables correlated with $r(t)$ and uncorrelated with $v(t)$ given by :

$$
\begin{aligned}
\zeta_{w}(t)=\left[r_{w}(t+l), r_{w}(t+l-1), \ldots r_{w}(t),\right. \\
\left.r_{w}(t-1), \ldots, r_{w}(t-l)\right]^{T}
\end{aligned}
$$

with $r_{w}(t)=W\left(q^{-1}\right) r(t)$ and $l$ a sufficiently large integer. The optimal controller parameters minimize the correlation criterion defined as the two-norm of the correlation function :

$$
\hat{\rho}=\arg \min _{\rho} J_{c}(\rho)=f^{T}(\rho) f(\rho)=\sum_{\tau=-l}^{l} R_{\varepsilon r_{w}}^{2}(\tau)
$$

where $R_{\varepsilon r_{w}}(\tau)$ is the cross-correlation function between the filtered reference signal $r_{w}(t)$ and $\varepsilon(\rho, t)$ defined by :

$$
\begin{aligned}
& R_{\varepsilon r_{w}}(\tau)=E\left\{\varepsilon(\rho, t) r_{w}(t-\tau)\right\} \\
& \quad=E\{[M-G(1-M) K(\rho)] r(t) W r(t-\tau)\}
\end{aligned}
$$

The correlation criterion can be represented in the frequency domain by applying Parseval's theorem when $l$ tends to infinity :

$$
\begin{aligned}
& \lim _{l \rightarrow \infty} J_{c}(\rho)=\frac{1}{2 \pi} \int_{-\pi}^{\pi}\left|\Phi_{\varepsilon r_{w}}(\omega)\right|^{2} d \omega \\
& =\frac{1}{2 \pi} \int_{-\pi}^{\pi}|W[M-G(1-M) K(\rho)]|^{2} \Phi_{r}^{2}(\omega) d \omega
\end{aligned}
$$

where $\Phi_{\varepsilon r_{w}}(\omega)$ is the cross-spectral density between $\varepsilon(\rho, t)$ and $r_{w}(t)$ and $\Phi_{r}(\omega)$ the spectrum of the reference signal.

\section{B. Filter design}

The weighting filter $W$ can be designed such that the correlation criterion (13) is equal to the model reference criterion (2). Replacing (3) in (2) and (13) gives :

$$
\begin{gathered}
J_{m r}(\rho)=\left\|\frac{\left[K^{*}-K(\rho)\right] G}{(1+K(\rho) G)\left(1+K^{*} G\right)}\right\|_{2}^{2} \\
\lim _{l \rightarrow \infty} J_{c}(\rho)=\frac{1}{2 \pi} \int_{-\pi}^{\pi} \frac{|W|^{2}|G|^{2}\left|K^{*}-K(\rho)\right|^{2}}{\left|1+K^{*} G\right|^{2}} \Phi_{r}^{2}(\omega) d \omega
\end{gathered}
$$

Comparing these two criteria, the following relation should hold for the weighting filter :

$$
\left|W\left(e^{-j \omega}\right)\right|^{2}=\frac{1}{\left|1+K\left(\hat{\rho}, e^{-j \omega}\right) G\left(e^{-j \omega}\right)\right|^{2} \Phi_{r}^{2}(\omega)}
$$

This filter depends on the controller to be designed $K(\hat{\rho})$ and the unknown plant $G$ and can therefore not be implemented. However, as proposed in [6], $K(\rho)$ can be approximated by $K^{*}$ to give :

$$
W\left(e^{-j \omega}\right)=\frac{1-M\left(e^{-j \omega}\right)}{\Phi_{r}(\omega)}
$$

This will lead to a very good approximation of the model reference criterion if the controller $K(\rho)$ is not under parameterized and the difference between $K^{*}$ and $K(\hat{\rho})$ is small.

\section{Tuning algorithm}

Let the controller be linearly parameterized in $\rho$ :

$$
K(\rho)=\beta^{T}\left(q^{-1}\right) \rho
$$

where $\beta\left(q^{-1}\right)$ is a vector of linear discrete-time transfer operators :

$$
\beta\left(q^{-1}\right)=\left[\beta_{1}\left(q^{-1}\right), \beta_{2}\left(q^{-1}\right), \ldots, \beta_{n_{\rho}}\left(q^{-1}\right)\right]^{T}
$$

with $n_{\rho}$ the number of controller parameters. Then, the error signal $\varepsilon(\rho, t)$ can be obtained by linear regression :

$$
\begin{aligned}
\varepsilon(\rho, t)=M\left(q^{-1}\right) r(t)-K(\rho)(1 & \left.-M\left(q^{-1}\right)\right) y(t) \\
& =y_{d}(t)-\phi^{T}(t) \rho
\end{aligned}
$$

where $y_{d}(t)=M\left(q^{-1}\right) r(t)$ and

$$
\phi(t)=\beta\left(q^{-1}\right)\left(1-M\left(q^{-1}\right)\right) y(t) .
$$




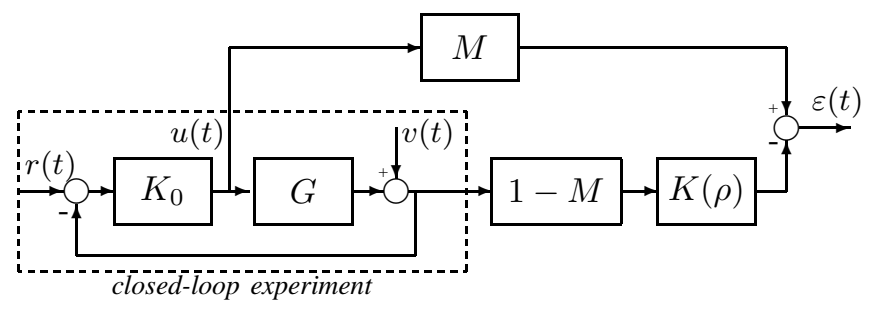

Fig. 4. Approximation of model reference control problem making use of a single closed-loop experiment

For a finite number of data $N$, the correlation function can be estimated as :

$\hat{f}(\rho)=\frac{1}{N} \sum_{t=1}^{N} \zeta_{w}(t) \varepsilon(t, \rho)=\frac{1}{N} \sum_{t=1}^{N} \zeta_{w}(t)\left[y_{d}(t)-\phi^{T}(t) \rho\right]$

Thus, the optimal controller parameters minimizing the correlation criterion $\hat{J}_{c}(\rho)=\hat{f}^{T}(\rho) \hat{f}(\rho)$ can be obtained by the standard-least squares algorithm :

$$
\hat{\rho}=\left(Q^{T} Q\right)^{-1} Q^{T} Z
$$

where

$$
Q=\frac{1}{N} \sum_{t=1}^{N} \zeta_{w}(t) \phi^{T}(t) \quad, \quad Z=\frac{1}{N} \sum_{t=1}^{N} \zeta_{w}(t) y_{d}(t)
$$

and $Q^{T} Q$ is nonsingular.

Note that $\hat{J}_{c}(\rho)$ converges to $J_{c}(\rho)$ when $N$ goes to infinity. However, for a finite number of data $\hat{J}_{c}(\rho)$ is biased. It can be shown that :

$$
E\left\{\hat{J}_{c}(\rho)\right\}=E\left\{\hat{f}^{T}(\rho) \hat{f}(\rho)\right\} \geqslant J_{c}(\rho)
$$

by considering the fact that $E\{\hat{f}(\rho)\}=f(\rho)$ and developing $E\left\{[\hat{f}(\rho)-f(\rho)]^{T}[\hat{f}(\rho)-f(\rho)]\right\} \geqslant 0$. It will be shown in Section $\mathrm{V}$ that the positive bias of the correlation criterion for a finite number of data helps improve the robustness of the controller.

\section{Closed-loop data}

The controller tuning scheme presented above can also be used when the data is collected in closed-loop operation with a stabilizing controller $K_{0}$. The set of data now consists of three signals, the reference signal $r(t)$ that is uncorrelated with the noise $v(t)$, and the measured input signal $u(t)$ and output signal $y(t)$ that are affected by $v(t)$. In the tuning scheme of Fig. 3, the plant is replaced by the closed-loop system, as shown in Fig. 4. Noting that :

$$
u(t)=\frac{K_{0}}{1+G K_{0}} r(t)-\frac{K_{0}}{1+G K_{0}} v(t)
$$

and

$$
y(t)=\frac{G K_{0}}{1+G K_{0}} r(t)+\frac{1}{1+G K_{0}} v(t),
$$

the error signal $\varepsilon(\rho, t)$ can be expressed as :

$$
\begin{aligned}
& \varepsilon(\rho, t)=M u(t)-(1-M) K(\rho) y(t) \\
& =\left[\frac{K_{0}}{1+G K_{0}} M-\frac{G K_{0}}{1+G K_{0}}(1-M) K(\rho)\right] r(t) \\
& -\left[\frac{K_{0}}{1+G K_{0}} M+\frac{1}{1+G K_{0}}(1-M) K(\rho)\right] v(t)
\end{aligned}
$$

As for the open-loop case, the correlation approach can be used to reduce the effect of noise on the criterion and the controller parameters. The error signal $\varepsilon\left(\rho^{*}, t\right)$ for the ideal controller $K^{*}=K\left(\rho^{*}\right)$ becomes the filtered noise signal, which will not be correlated with the reference signal $r(t)$ since $v(t)$ is not correlated with $r(t)$.

Using the vector of instrumental variables $\zeta_{w}(t)$ given by (10), the correlation criterion defined in (11) can be represented in the frequency domain as :

$$
\begin{aligned}
\lim _{l \rightarrow \infty} J_{c}(\rho)= & \frac{1}{2 \pi} \int_{-\pi}^{\pi}\left|\Phi_{\varepsilon r_{w}}(\omega)\right|^{2} d \omega \\
& =\frac{1}{2 \pi} \int_{-\pi}^{\pi} \mid W\left[\frac{K_{0}}{1+G K_{0}} M\right. \\
& \left.-\frac{K_{0}}{1+G K_{0}} G(1-M) K(\rho)\right]\left.\right|^{2} \Phi_{r}^{2}(\omega) d \omega
\end{aligned}
$$

Again, the filter $W$ can be designed such that the correlation criterion (26) is equal to the model reference criterion (2) :

$$
|W|^{2}=\frac{\left|1+G K_{0}\right|^{2}}{\left|K_{0}\right|^{2}|1+K(\hat{\rho}) G|^{2} \Phi_{r}^{2}(\omega)}
$$

This optimal filter depends on the unknown plant and the controller to be designed. It is now also dependent on the controller $K_{0}$ used in the experiment. Note that the power cross-spectral density function between the measured input $u(t)$ and $r(t)$ is :

$$
\Phi_{u r}(\omega)=\frac{K_{0}}{1+G K_{0}} \Phi_{r}(\omega) .
$$

Replacing $\Phi_{r}(\omega)$ in (27) by the expression obtained from (28) and using the same approximation as in the open-loop case (approximating $K(\rho)$ by $K^{*}$ ), the filter is now given by :

$$
W\left(e^{-j \omega}\right)=\frac{1-M\left(e^{-j \omega}\right)}{\Phi_{u r}(\omega)}
$$

The spectrum $\Phi_{u r}(\omega)$ can be estimated from the data by classical spectral analysis methods. Using the error signal defined in (25) and the filter (29), the algorithm presented in III-C can also be used when the data is collected in closedloop operation and both the plant input $u(t)$ and the plant output $y(t)$ are affected by noise.

\section{Controller Validation}

The main difficulty with data-driven controller tuning methods is the stability of the resulting system. Data-driven methods as Virtual Reference Feedback Tuning (VRFT), Iterative Feedback Tuning (IFT) and Correlation based Tuning $(\mathrm{CbT})$ do not explicitly address this issue, and the stability of 


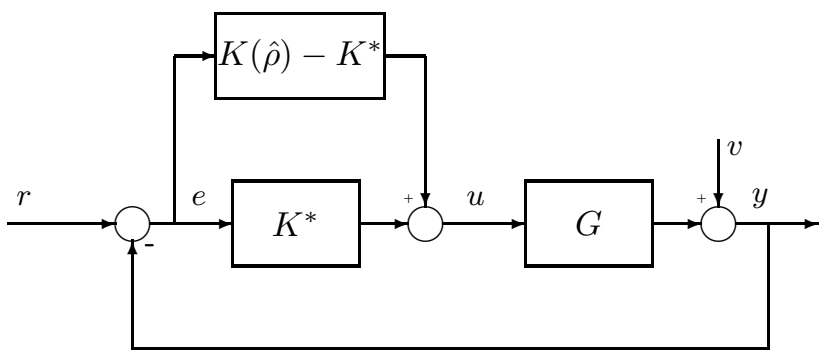

Fig. 5. Stability Condition

the system after implementation of the controller cannot be guaranteed. Some attempts to improve the robustness of the tuned controller have been reported for the IFT method in [9], [10]. A model-based approach is used to check the stability before implementation of the controller in [11] for VRFT and in [4] for CbT. However, this needs the identification of a reliable plant model which is not always an easy task.

In Iterative Controller Unfalsification (ICU), a data-driven test is proposed to check the performance objective in terms of the infinity norm of a closed-loop transfer function. The main problem in this approach is the effect of noise. The noise is characterized as a two-norm bounded sequence, i.e. without stochastic properties. Hence, there is no restriction preventing correlation between noise and input and this typically leads to underestimation of the infinity norm. To improve the results, [12] introduced a low-correlated bounded noise set by using a constraint on the mean value of the noise sequence.

In this section, a new validation test based on the correlation approach is introduced to evaluate the stability of the closed-loop system. This test can be applied to any controller designed for model reference control problem before applying it to the real plant and requires a single set of data.

\section{A. Data-driven stability test}

The objective is to evaluate the stability of controller $K(\hat{\rho})$ in closed loop with the real plant $G$. This closed-loop system can be represented by interconnection of the reference model and the difference between the ideal controller $K^{*}$ and the computed one (see Fig. 5). Based on the small gain theorem, a sufficient condition for the stability of this closed-loop system is :

$$
\delta=\left\|\left(-K(\hat{\rho})+K^{*}\right) G(1-M)\right\|_{\infty}<1
$$

Replacing $K^{*} G(1-M)$ by $M$ gives :

$$
\delta=\|M-K(\hat{\rho}) G(1-M)\|_{\infty}<1
$$

This transfer function is exactly the transfer function between $r(t)$ and $\varepsilon(\hat{\rho}, t)$ in the tuning scheme of Fig. 3. The infinity norm $\delta$ of this transfer function can be computed by identification of an input-output model in the time or frequency domains. Here, a direct data-driven approach to estimate the infinity norm of a transfer function is used since it does not require any modeling of the system. This approach has been used for time-domain validation in the context of robust identification [13] and controller unfalsification [7]. In this paper, the results of this approach are improved in the presence of noise by introducing some new constraints based on the decorrelation of the noise and the reference signal.

To proceed, consider the $(N+m-1, m)$ Toeplitz matrix

$$
T_{m}(r)=\left[\begin{array}{cccc}
r(1) & 0 & . . & 0 \\
r(2) & r(1) & . . & 0 \\
: & r(2) & . . & 0 \\
r(N) & : & . . & r(1) \\
0 & r(N) & . . & r(2) \\
: & 0 & . . & : \\
0 & : & . . & r(N)
\end{array}\right]
$$

Then as $N$ and $m$ go to infinity and $m / N$ goes to zero :

$$
\lambda_{\max }\left(\frac{1}{N} T_{m}(r)^{T} T_{m}(r)\right) \nearrow \sup _{\omega} \Phi_{r}(\omega)
$$

where $\lambda_{\max }(\cdot)$ denotes the maximum eigenvalue and $\nearrow$ the convergence from below [14]. Now, suppose that noise-free data are collected, that is :

$$
\varepsilon(\hat{\rho}, t)=[M-K(\hat{\rho}) G(1-M)] r(t)
$$

An estimate of $\delta$ can be obtained as the solution to the following convex optimization problem :

$$
\begin{aligned}
& \qquad \hat{\delta}=\min _{\delta} \delta \\
& \text { subject to : } \\
& T_{m}^{T}(\varepsilon(\hat{\rho})) T_{m}(\varepsilon(\hat{\rho}))-\delta^{2} T_{m}^{T}(r) T_{m}(r) \leqslant 0
\end{aligned}
$$

For large $N$ and $m$, and small $m / N$, one has :

$$
\hat{\delta}^{2} \approx \max _{\left\{\omega \mid \Phi_{r}(\omega) \neq 0\right\}} \frac{\Phi_{\varepsilon}(\omega)}{\Phi_{r}(\omega)}
$$

It can be shown that $\hat{\delta}$ converges to $\delta$ when $N$ and $m$ go to infinity, $m / N$ goes to zero and $\Phi_{r}(\omega)>0, \forall \omega \in \mathcal{R}$ [14].

In the presence of noise, the error signal is :

$$
\varepsilon(\hat{\rho}, t)=[M-K(\hat{\rho}) G(1-M)] r(t)-K(\hat{\rho})(1-M) v(t)
$$

It is suggested in [7] to introduce a new variable $w(t)$ for $t=1, \ldots, N$ to compensate the effect of noise. The convex optimization problem in this case becomes :

$$
\hat{\delta}=\min _{\delta, w} \delta
$$

subject to :

$$
\begin{aligned}
& T_{m}^{T}\left(\varepsilon_{w}(\hat{\rho})\right) T_{m}\left(\varepsilon_{w}(\hat{\rho})\right)-\delta^{2} T_{m}^{T}(r) T_{m}(r) \leqslant 0 \\
& \frac{1}{N} \sum_{t=1}^{N} w^{2}(t) \leqslant \sigma^{2}
\end{aligned}
$$

where

$$
\varepsilon_{w}(\hat{\rho}, t)=\varepsilon(\hat{\rho}, t)+K(\hat{\rho})(1-M) w(t)
$$

and $\sigma^{2}$ is the variance of $v(t)$ that is supposed to be known. It should be mentioned that $\delta$ in this case does not converge to $\delta$ even for an infinite number of data. The main reason is that 
there is an infinite number of sequences $w(t)$ that satisfy the norm bound in (37) but do not meet the stochastic properties of $v(t)$, in particular the fact that $w(t)$ like $v(t)$ should not be correlated with the reference signal $r(t)$. Here, we suggest to add two convex constraints to the optimization problem (37) that take into account the fact that $r(t)$ and $w(t)$ are uncorrelated. This will significantly improve the estimation of the infinity norm from noisy data.

\section{B. Correlation-based stability test}

Let the instrumental variable vector $\zeta(t)$ be defined as :

$$
\zeta(t)=[r(t+l), \cdots, r(t), \cdots, r(t-l)]^{T}
$$

and the decorrelation criterion $J_{N}(w)$ as :

$$
\begin{aligned}
J_{N}(w) & =\left[\frac{1}{N} \sum_{t=1}^{N} w(t) \zeta(t)\right]^{T}\left[\frac{1}{N} \sum_{t=1}^{N} w(t) \zeta(t)\right] \\
& =\sum_{\tau=-l}^{l} \hat{R}_{w r}^{2}(\tau)
\end{aligned}
$$

where

$$
\hat{R}_{w r}(\tau)=\frac{1}{N} \sum_{t=1}^{N} w(t) r(t-\tau)
$$

If $w(t)$ is considered as a realization of a random variable independent of $r(t)$, then $\sqrt{N} \hat{R}_{w r}(\tau)$ tends in distribution to a normal distribution with zero-mean and variance $P$ given by [15] :

$$
P=\sum_{\tau=-\infty}^{\infty} R_{w}(\tau) R_{r}(\tau)
$$

where $R_{w}(\tau)$ and $R_{r}(\tau)$ are the auto-correlation functions of $w(t)$ and $r(t)$, respectively. When $r(t)$ is white, the variance reduces to $P=\sigma^{2} R_{r}(0)$. Moreover, $J_{N}(w)$ has a chi-square distribution with variance $P$. The confidence intervals for $\hat{R}_{w r}(\tau)$ and $J_{N}(w)$ are thus given by :

$$
\begin{gathered}
\left|\hat{R}_{w r}(\tau)\right| \leqslant \sqrt{\frac{P}{N}} \mathcal{N}_{\alpha} \quad \text { for } \tau=-l, \ldots, l \\
J_{N}(w) \leqslant \frac{P}{N} \chi_{\alpha}^{2}(2 l+1)
\end{gathered}
$$

where $\mathcal{N}_{\alpha}$ denotes the $\alpha$-level of the unit variance normal distribution and $\chi_{\alpha}^{2}(2 l+1)$ the $\alpha$-level chi-square distribution with $2 l+1$ degree of freedom. These convex constraints can be added to the optimization problem in (37) to improve the estimate of $\delta$. This will be illustrated with the following simulation example.

Example: Consider a discrete-time oscillatory system with the pair of complex poles $0.1+0.3 j$ and $0.1-0.3 j$, a steady state gain of 1 and the sampling time $T_{s}=0.05 \mathrm{~s}$. This system has an $H_{\infty}$ norm of 1.167. A set of input/output data is generated using a PRBS input signal of several periods, each of length 511 with amplitude 0.1 . The output is disturbed by a white noise with a standard deviation of $\sigma=0.0315$. The estimate $\hat{\delta}$ of the infinity norm of the system is calculated for different data length $N$ of the given input/output sequences, once using the optimization problem (37) and a second time adding the correlation-based constraints (42) and (43). The variance $\sigma^{2}$ of $w(t)$ is fixed to the same value as the real noise signal $v(t)$. The results using an increasing number of data are shown in Fig. 6. The ratio $N / m$ is fixed to 40 and $l$ to 15 for an $\alpha$-level of 0.95 .

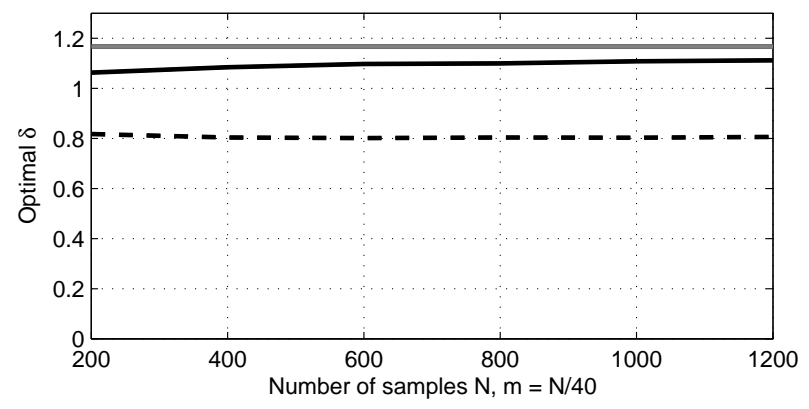

Fig. 6. $\hat{\delta}$ found assuming $\|v(t)\|_{2}^{2} \leqslant \sigma^{2}$ (thick dashed line), $\hat{\delta}$ found using the correlation constraints (thick line) and the $H_{\infty}$ norm of the system (grey line)

Without the correlation constraints, $\delta$ is highly underestimated and increasing the number of data does not affect this underestimation. When adding the correlation constraints, the underestimation of $\delta$ for small data lengths is about $9 \%$. Increasing the number of data improves the result, for $N=1200$ the underestimation is less than $5 \%$.

\section{Simulation EXAMPLE}

Consider the flexible transmission system proposed in [16] as a benchmark for digital control design. This example was used by Campi et al. in [6] to demonstrate the VRFT approach and also by Sala and Esparza in [11]. In this comparative simulation example, the same plant $G\left(q^{-1}\right)$, reference model $M\left(q^{-1}\right)$ and basis function $\beta\left(q^{-1}\right)$ as in [6] are used; furthermore, similar data length, input signal characteristics and signal-to-noise ratio are used.

The plant is described by the discrete-time model $G\left(q^{-1}\right)$ with the sampling time $T_{s}=0.05 \mathrm{~s}$

$$
G\left(q^{-1}\right)=\frac{0.28261 q^{-3}+0.50666 q^{-4}}{A\left(q^{-1}\right)}
$$

with

$$
\begin{aligned}
A\left(q^{-1}\right)=1- & 1.41833 q^{-1}+1.58939 q^{-2} \\
- & 1.31608 q^{-3}+0.88642 q^{-4}
\end{aligned}
$$

The control objective is defined by the closed-loop reference model

$$
M\left(q^{-1}\right)=\frac{q^{-3}(1-\alpha)^{2}}{\left(1-\alpha q^{-1}\right)^{2}}, \quad \alpha=e^{-T_{s} \bar{\omega}}, \quad \bar{\omega}=10,
$$

where $\bar{\omega}$ is the desired bandwidth. The controller structure is defined as

$$
K(\rho)=\frac{\rho_{0}+\rho_{1} q^{-1}+\rho_{2} q^{-2}+\rho_{3} q^{-3}+\rho_{4} q^{-4}+\rho_{5} q^{-5}}{1-q^{-1}}
$$


The performance and the characteristics of the proposed method will be illustrated using the above plant and reference model. A comparison with the VRFT approach will be given in terms of performance and and sensitivity to noise.

\section{A. Control Performance and Sensitivity to Noise}

In order to compute a controller using the VRFT method, two sets of data are required. The data are generated using a 511-sample PRBS signal with amplitude 0.1. The output is disturbed by zero-mean white noise such that the signalto-noise ratio at the output of the plant is about 10 in terms of variance. Since the proposed method uses a single set of input/output data, another set of open-loop input/output data is generated using two periods of the PRBS signal used for the VRFT, i.e. with $N=1022$. The output is disturbed by the same noise realization as the output for the VRFT. The length of the instrumental variable vector (10) is $l=35$, which corresponds to the length of the impulse response of $M$.

Using the data sets as explained above, two controllers are calculated. The estimated controller parameter vector for the proposed approach is :

$$
\hat{\rho}=\left[\begin{array}{lrrr}
0.2315 & -0.3286 & 0.3192 & -0.261 \\
& 0.2107 & -0.03225
\end{array}\right]^{T}
$$

The achieved performance of this controller in terms of the original model reference criterion (2) is $J_{m r}=0.1083$. The parameters of the VRFT controller are :

$$
\hat{\rho}_{V R F T}=\left[\begin{array}{llll}
0.2383 & -0.3387 & 0.3261 & -0.2547 \\
0.1991 & -0.02382
\end{array}\right]^{T}
$$

and the achieved performance is $J_{m r}=0.1077$. The magnitude bode plots of the controlled systems are shown in Fig.7.

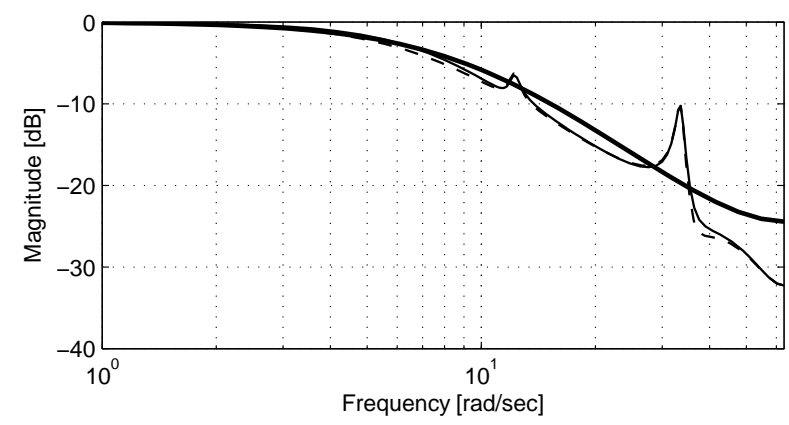

Fig. 7. Magnitude Bode plots, $M$ (thick line), System controlled by VRFT controller (thin line) and System controlled by proposed controller (dashed line)

The performance of the proposed method is comparable to that of the VRFT method. However, the effect of noise is different as is shown by a Monte Carlo simulation with 100 experiments. Out of 100 VRFT controllers, 10 are destabilizing, whereas all the controllers calculated by the proposed method are stabilizing the closed-loop system. Among the remaining 90 VRFT controllers, 58 achieve a $J_{m r}<0.15$. For 7 stabilizing controllers, the achieved $J_{m r}$ is greater than 0.5 . For the 100 controllers computed by the proposed method, the mean of $J_{m r}$ is 0.1101 and the sample variance is about $10^{-4}$.

Although, the variance of the controllers designed using the proposed method is low, the finite data length estimates are biased. This can be seen in Fig. 8. The high-frequency gain achieved by all controllers is lower than the estimate found using noise-free data. The bias decreases the controller gain at frequencies where the sensitivity $1-M$ is high, which improves the stability of the controlled system. The stabilizing effect of the bias in this simulation example can be expected in the general case as a bias analysis of the correlation criterion $\hat{J}_{c}(\rho)$ for finite data length would show.

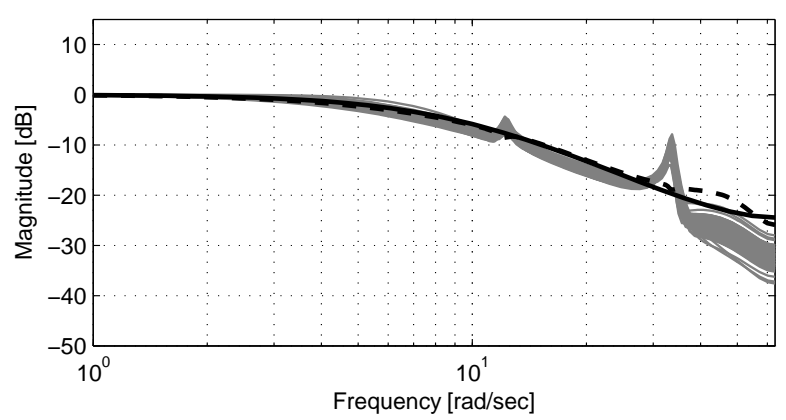

Fig. 8. Magnitude Bode plots of $M$ (thick line), achieved closed-loop systems in the Monte Carlo simulation (grey lines) and in the noise-free case (dashed line) for the proposed method

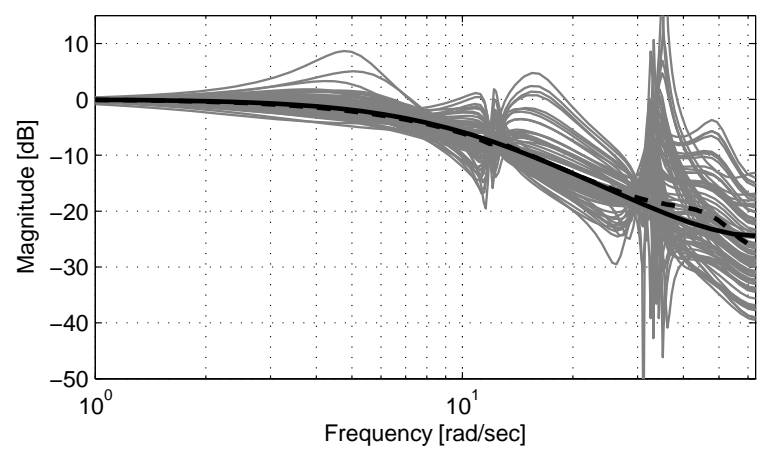

Fig. 9. Magnitude Bode plots of $M$ (thick line), achieved closed-loop systems in the Monte Carlo simulation (grey lines) and in the noise-free case (dashed line) for the VRFT method (for only 90 stabilizing controllers)

\section{B. Stability test with the various controllers}

In the simulation example discussed above, 200 controllers for the flexible transmission system are computed, 100 using the VRFT approach and 100 using the proposed approach. Both methods are data-driven and do not guarantee stability of the closed-loop system. All 200 controllers are subjected to the data-driven controller validation test presented in Section IV. 
The same data as used to compute the controllers is available for controller validation. $N=511$ samples are used in the test, $m$ and $l$ are taken equal to 50, $\sigma$ equal to the standard deviation of the noise in the data and $\alpha=0.9$.

The proposed tuning method leads to 100 stabilizing controllers out of 100 which are all have a $\delta$ less than 1. All controllers are thus validated with the estimated value of the infinity norm by the optimization problem (37) with or without using the correlation constraints (42) and (43). This was expected because the optimization problem underestimates the infinity norm of the transfer functions. If no noise vector is considered in the optimization problem, an upper bound for $\delta$ will be estimated. The use of this upper bound for controller validation reduces significantly the risk of validation of destabilizing controllers. This test, however, invalidates 10 stabilizing controllers.

The VRFT approach gives 90 stabilizing controllers, among which 16 have $\delta>1$. The infinity-norm estimates by the optimization problem (37) without the correlation constraints invalidate only 6 out of the 10 destabilizing controllers. The correlation-based stability test invalidates 9 out of the 10 destabilizing VRFT controllers. Using the upper bound of the infinity norm leads to invalidation of 58 of the VRFT controllers, among which 48 stabilize the true system and 32 have $\delta<1$.

\section{CONCLUSIONS}

A noniterative correlation-based data-driven algorithm for tuning fixed-order controllers is proposed. A correlation criterion is defined that is convex with respect to linearly parameterized controllers and approximates the model reference control criterion. An estimate of the correlation criterion is minimized by the standard least-squares algorithm. Although this estimate converges asymptotically to the correlation criterion, it is biased for a finite number of data. A simulation example shows that the biasedness of the criterion estimate improves the robustness of the tuned controller. However, for the general case, a non-asymptotic bias analysis of the estimate could be performed in the frequency-domain.

A new data-driven stability test is proposed that can be applied to any controller designed for the model reference control problem. The closed-loop stability is guaranteed if the infinity norm of a transfer function is less than 1. An estimate of this infinity norm is obtained as the solution to a convex optimization problem. It is shown that the estimate of the infinity norm is improved by adding to the optimization problem some convex constraints representing the independence of noise and reference signal. The conditions for asymptotic convergence of the estimate in the presence of noise need to be investigated.

\section{REFERENCES}

[1] A. M. Veres and D. S. Wall, Synergy and Duality of Identification and Control. London, UK: Taylor and Francis, 2000.

[2] H. Hjalmarsson, M. Gevers, S. Gunnarsson, and O. Lequin, "Iterative feedback tuning: Theory and application," IEEE Control Systems Magazine, pp. 26-41, 1998.
[3] H. Hjalmarsson, "Iterative feedback tuning - an overview," International Journal of Adaptive Control and Signal Processing, vol. 16, pp. 373-395, 2002.

[4] A. Karimi, L. Mišković, and D. Bonvin, "Iterative correlation-based controller tuning," International Journal of Adaptive Control and Signal Processing, vol. 18, no. 8, pp. 645-664, 2004.

[5] G. O. Guardabbassi and S. M. Savaresi, "Virtual reference direct design method: an off-line approach to data-based control system design," IEEE Transactions on Automatic Control, vol. 45, no. 5, pp. 954-959, 2000.

[6] M. C. Campi, A. Lecchini, and S. M. Savaresi, "Virtual reference feedback tuning: a direct method for the design of feedback controllers," Automatica, vol. 38, pp. 1337-1346, 2002.

[7] R. L. Kosut, "Uncertainty model unfalsification for robust adaptive control," Annual Reviews in Control, vol. 25, pp. 65-76, 2001.

[8] M. C. Campi and S. M. Savaresi, "Direct nonlinear control design: The virtual reference feedback tuning (VRFT) approach," IEEE Transactions on Automatic Control, vol. 51, no. 1, pp. 14-27, 2006.

[9] H. Hjalmarsson and S. M. Veres, "Robust loopshaping using iterative feedback tuning," in European Control Conference, Porto, Portugal, September 2001, pp. 2046-2051.

[10] H. Prochazka, M. Gevers, B. Anderson, and C. Ferrera, "Iterative feedback tuning for robust controller design and optimization," in 44th IEEE Conference on Decision and Control and European Control Conference, 2005, pp. 3602-3607.

[11] A. Sala and A. Esparza, "Extensions to "virtual reference feedback tuning: A direct method for the design of feedback controllers"," Automatica, vol. 41, no. 8, pp. 1473-1476, 2005.

[12] H. Fukushimai and T. Sugie, "Identification of unfalsified plant model sets based on low-correlated bounded noise model," in 38th IEEE Conference on Decision and Control, vol. 5, 1999, pp. 5088-5093.

[13] K. Poolla, P. Khargonekar, A. Tikku, J. Krause, and K. Nagpal, "A time-domain approach to model validation," IEEE Transactions on Automatic Control, vol. 39, no. 5, pp. 951-959, May 1994.

[14] M. Massoumnia and R. L. Kosut, "A family of norms for system identification problems," IEEE Transactions on Automatic Control, vol. 39, no. 5, pp. 1027-1031, 1994.

[15] L. Ljung, System Identification - Theory for the User. NJ, USA: Prentice Hall, 1999.

[16] I. D. Landau, D. Rey, A. Karimi, A. Voda, and A. Franco, "A flexible transmission system as a benchmark for robust digital control," European Journal of Control, vol. 1, no. 2, pp. 77-96, 1995. 